\title{
SOME CLASSES OF ALPHA-QUASI-CONVEX FUNCTIONS
}

\author{
KHALIDA INAYAT NOOR \\ Mathematics Department \\ College of Science Education for Girls, Sitteen Road, \\ Malaz, Riyadh, Saudi Arabia.
}

(Received April 13, 1985 and in revised form May 30, 1986)

ABSTRACT. Let $C[C, D],-1 \leq D<C \leq 1$ denote the class of functions $g, g(0)=0$ $g^{\prime}(0)=1$, analytic in the unit disk $E$ such that $\frac{\left(\mathrm{zg}^{\prime}(z)\right)^{\prime}}{g^{\prime}(z)}$ is subordinate to $\frac{1+C Z}{1+D Z}, z \varepsilon E$. We investigate some classes of Alpha-Quasi-Convex Functions $f$, with $f(0)=f^{\prime}(0)-l=0$ for which there exists a geC [C,D] such that $(1-\alpha) \frac{f^{\prime}(z)}{g^{\prime}(z)}+\alpha \frac{\left(z f^{\prime}(z)\right)^{\prime}}{g^{\prime}(z)}$ is subordinate to $\frac{1+A Z}{1+B Z},-1 \leq B<A \leq 1$. Integral representation, coefficient bounds are obtained. It is shown that some of these classes are preserved under certain integral operators.

KEY WORDS AND PHRASES. Convex, starlike, quasi-convex, close-to-convex function, Integral representation, Alpha-quasi-convex functions. AMS (MOS) Subject classification (1980) Codes: $30 c 45,30 C 55$.

\section{INTRODUCTION}

$$
{ }_{\infty} \text { et } S, K, S^{*} \text { and } C \text { denote the classes of analytic functions } f:
$$
$f(z)=z+\sum a_{n} z^{n}$ which are respectively univalent, close-to-convex, starlike (with $=2$ respect to the origin) and convex in the unit disc $E$. In [1], a new subclass $C^{*}$ of univalent functions was introduced and studied. A function $f$ belongs to $C^{*}$ if there exists a convex function $g$ such that, for $z \varepsilon E$,

$$
\operatorname{Re} \frac{\left(z f^{\prime}(z)\right)^{\prime}}{g^{\prime}(z)}>0
$$

The functions in $C^{*}$ are called quasi-convex functions and $C C^{*} C K C S$. It is also sknown that $f \varepsilon C^{*}$, if, and only if, zf' $\varepsilon$. For complete study of $C^{*}$, see Noor [2].

A new class $Q_{\alpha}$ of $\alpha$-quasi-convex functions has been defined and discussed in some details in [3]. A function $f$ belongs to the class $Q_{\alpha}, \alpha$ real, if and only if there exists a convex function g such that, for $z \varepsilon E$

$$
\operatorname{Re}\left[(1-\alpha) \frac{f^{\prime}(z)}{g^{\prime}(z)}+\alpha \frac{\left(z f^{\prime}(z)\right)^{\prime}}{g^{\prime}(z)}\right]>0
$$

We note that $Q_{0}=k$ and $Q_{1}=C^{*}$.

In [4], Janowski introduced the calss $P[A, B]$. For $A$ and $B, 1 \leq B \leq A \leq 1$, a function $p$, analytic in $E$ with $p(0)=1$ belongs ro the $\operatorname{class} P[A, B], i f p(z)$ is subordinate to $\frac{1+A Z}{1+B Z}$. Also, given $C$ and $D,-1 \leq D<C \leq 1, C[C, D]$ and $S^{*}[C, D]$ 
denote the classes of functions $f$ analytic in $E$ with $f(z)=z+\sum_{n=2}^{\infty} a_{n} z^{n}$ such that $\frac{\left(z f^{\prime}(z)\right)^{\prime}}{f^{\prime}(z)} \varepsilon P[C, D]$ and $\frac{z f^{\prime}(z)}{f(z)} \varepsilon P[C, D]$ respectively. For $C=1$ and $D=-1$ we note that $C[1,-1]=C$ and $S^{\star}[1,-1]=S^{\star}$. Silvia $[5]$ defines the classes $K[A, B ; C, D]$ as follows:

Definition 1.1. A function $f: f(z)=z+\sum_{n=2}^{\infty} a_{n} z^{n}$, analytic in E, is said to be in the class $K[A, B ; C, D],-1 \leq B \leq A \leq 1 ; n=2 \leq D \leq C \leq 1$, if there exists a $\mathrm{g} \varepsilon \mathrm{C}[\mathrm{C}, \mathrm{D}]$ such that $\frac{\mathrm{f}^{\prime}(\mathrm{z})}{\mathrm{g}^{\prime}(\mathrm{z})} \varepsilon P[\mathrm{~A}, \mathrm{~B}]$.

It is clear that $\mathrm{K}[1,-1 ; 1,-1]=\mathrm{K}$ and

$$
\mathrm{K}[\mathrm{A}, \mathrm{B} ; \mathrm{C}, \mathrm{D}] \subset \mathrm{K} C \mathrm{~S} \text {. }
$$
We now define the following:
Definition 1.2 . Let $\alpha \geq 0$ be real and $f: f(z)=z+\sum_{n=2}^{\infty} a_{n} z^{n}$ be analytic in $E$. Then $f \in Q_{\alpha}[A, B ; C, D],-1 \leq B<A \leq 1 ;-1 \leq D<C \leq 1$ if and only if there exists a function $g \in C[C, D]$ such that, for $z \varepsilon E$,

$$
(1-\alpha) \frac{f^{\prime}(z)}{g^{\prime}(z)}+\alpha \frac{\left(z f^{\prime}(z)\right)^{\prime}}{g^{\prime}(z)} \varepsilon P[A, B] \text {. }
$$

It is clear that $Q_{\alpha}[1,-1 ; 1,-1]=Q_{\alpha}$.

\section{MAIN RESULTS}

We shall now study some of the basic properties of the class $Q_{\alpha}[A, B ; C, D]$. From the definition 1.2 , we immediately have: THEOREM 2.1. Let $F(z)=(1-\alpha) f(z)+\alpha z f^{\prime}(z)$, where $0<\alpha<1$ is real and $z \varepsilon E$. Then $f \in Q_{\alpha}[A, B ; C, D],-1 \leq B<A \leq 1 ;-1 \leq D<C \leq 1$ if and only if $F \in K[A, B ; C, D]$.

We now give the integral representation for the functions in the class $\&_{\alpha}[A, B ; C, D]$.

THEOREM 2.2. A function $f \in Q_{\alpha}[A, B ; C, D]$, for $\alpha>0,-1 \leq B<A \leq 1 ;-1 \leq D<C \leq 1$, if and only if there exists a function $F \varepsilon K[A, B ; C, D]$ such that, for $z \varepsilon E$,

$$
f(z)=\frac{1}{\alpha} z^{1-\frac{1}{\alpha}} \int_{0}^{z} \zeta^{\frac{1}{\alpha}-2} F(\zeta) d \zeta
$$

PROOF. From (2.1), it follows that

$$
\left(\frac{1}{\alpha}-1\right) z^{\frac{1}{\alpha}-2} f(z)+\alpha z^{\frac{1}{\alpha}-1} f^{\prime}(z)=z^{\frac{1}{\alpha}-2} F(z),
$$

so

$$
(1-\alpha) f(z)+\alpha z f^{\prime}(z)=F(z)
$$

and the result follows immediately from theorem 2.1 .

THEOREM 2.3. Let $f \in Q_{\alpha}[\mathrm{A}, \mathrm{B} ; \mathrm{C}, \mathrm{D}], 0<\alpha<1$ and $-1 \leq \mathrm{B}<\mathrm{A} \leq 1 ;-1 \leq \mathrm{D}<\mathrm{C} \leq 1$. Then $\mathrm{f} \varepsilon \mathrm{K}[\mathrm{A}, \mathrm{B} ; \mathrm{C}, \mathrm{D}]$ and hence is univalent.

PROOF. Silvia [5] has proved that if $f_{1} \varepsilon K[A, B ; C, D]$, then so is

$$
F_{1}(z)=\frac{1+\gamma_{1}}{\gamma_{1}} \int_{0}^{z} t \gamma_{1}^{-1} f_{1}(t) d t, \text { Re } \gamma_{1}>0 \text {. }
$$

Using this result and the integral representation (2.2) with $\gamma_{1}=\frac{1}{\alpha}-1$ for $\mathrm{ft}_{\mathrm{r}}[\mathrm{A}, \mathrm{B} ; \mathrm{C}, \mathrm{D}]$, we obtain the required result. 
For our next theorem, we need the following result due to silvia [5]. LEMMA 2.1. Let $F \in K[A, B ; C, D]$ and $F(z)=z+\sum_{n=2}^{\infty} b_{n} z^{n}$. Then

$$
\left|b_{2}\right| \leq \frac{(C-D)+(A-B)}{2} \text {, }
$$

and

$$
\left|b_{3}\right| \leq\left\{\begin{array}{l}
\frac{C-D}{6}+\frac{(A-B)(C-D+1)}{3},|C-2 D| \leq 1 \\
\frac{(C-D)(C-2 D)}{6}+\frac{(A-B)(C-D+1)}{3},|C-2 D|>1 .
\end{array}\right.
$$

THEOREM 2.4. Let $F \in Q_{\alpha}[A, B ; C, D], 0<\alpha<1$ and $f(z)=z+\sum_{n=2}^{\infty} a_{n} z^{n}$. Then

$$
\left|a_{2}\right| \leq \frac{1}{1+\alpha}\left[\frac{(C-D)+(A-B)}{2}\right],
$$

and

$$
\left|a_{3}\right| \leq \frac{1}{(1+2 \alpha)}\left[\begin{array}{l}
\left.\frac{(C-D)}{6}+\frac{(A-B)}{3}, \mid C-D+1\right) \\
\frac{(C-D)(C-2 D)}{2}+\frac{(A-B)(C-D+1)}{3},|(C-2 D)|>1
\end{array}\right.
$$

PROOF. Since $f \varepsilon Q_{\alpha}[A, B ; C, D]$, by theorem 2.1 , the function

$$
F(z)=(1-\alpha) f(z)+\alpha z f^{\prime}(z)
$$

belongs to $K[A, B ; C, D]$. Let $F(z)=z+\sum_{n=2}^{\infty} b_{n} z^{n}$.

Thus

$$
(1-\alpha)\left[z+\sum_{n=2}^{\infty} a_{n} z^{n}\right]+\alpha z\left[1+\sum_{n=2}^{\infty} n a_{n} z^{n}\right]=z+\sum_{n=2}^{\infty} b^{b} z^{n}
$$

or

$$
\text { (1- } 1) \sum_{n=2}^{\infty} a_{n} z^{n}+\alpha \sum_{n=2}^{\infty} n a_{n} z^{n}=\sum_{n=2}^{\infty} b_{n} z^{n} .
$$

Equating coefficients of $z^{n}$ on both sides, we have

$$
[(1-\alpha)+\alpha n] a_{n}=b_{n}
$$

Now, using Lemma 2.1 and the relation (2.3), we obtain the required result. REMARK 2.1. Let FEK $[A, B ; 1,-1]$ and be given by $F(z)=z+\sum_{n=2}^{\infty} b_{n} z^{n}$. Then

$$
\left|b_{2}\right| \leq \frac{1}{2}(A-B+2) \text {. }
$$

This result is sharp for the function $F_{0} \in K[A, B, 1,-1]$ and defined by

$$
F_{0}(z)=\int_{0}^{z} \frac{(1+A w)}{(1-w)^{2}(1+B w)} d w .
$$

3. THE CLASS $Q_{\alpha}[1-2 \beta,-1 ; 1-2 \gamma,-1]$

In definition 1.2 , if we put $A=1-2 \beta, B=-1 ; C=1-2 \gamma, D=-1$, then we have the following:

Definition 3.1. A function $f$, analytic in $E$, is said to be alpha-quasiconvex of order $\beta$ type $\gamma$, if, and only if, there exists a function 
$g \in C[1-2 \gamma,-1]$ such that

$$
H(\alpha, f)=(1-\alpha) \frac{f^{\prime}(z)}{g^{\prime}(z)}+\alpha \frac{\left(z f^{\prime}(z)\right)}{g^{\prime}(z)} \varepsilon_{\varepsilon} P[1-2 \beta,-1]
$$

REMARK 3.1. Let $g$ be analytic in $E$. Then $g \in C[1-2 \gamma,-1]$ if and only if

$$
\operatorname{Re} \frac{\left(z g^{\prime}(z)\right)^{\prime}}{g^{\prime}(z)}>\gamma, \quad z \in E \text {. }
$$

Thus $H(\alpha, f) \in P[1-2 \beta,-1]$ implies that

$$
\operatorname{Re}\left[(1-\alpha) \frac{f^{\prime}(z)}{g^{\prime}(z)}+\alpha \frac{\left(z f^{\prime}(z)\right)^{\prime}}{g^{\prime}}\right]>\beta, \quad z \in E .
$$

REMARK 3.2. It follows, from the definition 3.1 , that $f \in Q_{\alpha}[1-2 \beta,-1 ; 1-2 \gamma,-1]$ if, and only if $\left\{(1-\alpha) f+\alpha z f^{\prime}\right\} \in K[1-2 \beta,-1 ; 1-2 \gamma,-1]$.

We now have the following:

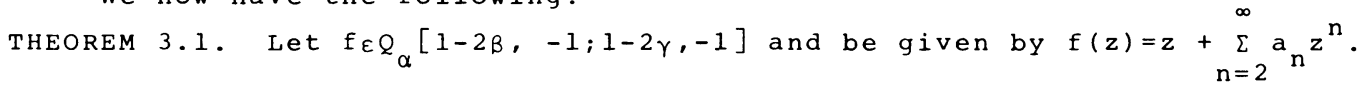
Then we have, for $n \geq 2$

$$
\left|a_{n}\right| \leq \frac{2(3-2 \gamma)(4-2 \gamma) \ldots \ldots(n-2 \gamma)[n(1-\beta)+\beta-\gamma]}{n ![1+\alpha(n-1)]} .
$$

This result is sharp and the equality holds for the function $f_{0}$ defined as

$$
f_{0}(z)= \begin{cases}\frac{1}{\alpha} z^{1-\frac{1}{\alpha}} \int_{0}^{z}\left(\frac{1}{\alpha}-2\right) & \left(\zeta(1-\gamma)(1-2 \beta)+(\beta-\gamma)\left[1-(1-\zeta)^{2-2 \gamma}\right]\right) d \zeta, \gamma \neq 1, \gamma \neq \frac{1}{2} \\ \frac{1}{\alpha} z^{1-\frac{1}{\alpha}} \int_{0}^{z} \frac{1}{\frac{1}{\alpha}-2} & {\left[(1-2 \beta) \log (1-\zeta)+\frac{2(1-\beta) \zeta}{1-\zeta}\right] d \zeta, \quad \gamma=\frac{1}{2}} \\ \frac{1}{\alpha} z^{1-\frac{1}{\alpha}} \int_{0}^{z} \zeta_{0}^{\frac{1}{\alpha}-2} & {[2(\beta-1) \log (1-\zeta)+(2 \beta-1) \zeta] d \zeta, \quad \gamma=1}\end{cases}
$$

PROOF. Since $f \varepsilon Q_{\alpha}[1-2 \beta,-1 ; 1-2 \gamma,-1]$, the function

$$
F(z)=(1-\alpha) f(z)+\alpha z f^{\prime}(z){ }_{\infty}
$$

belong to $k[1-2 \beta,-1 ; 1-2 \gamma,-1]$. Let $F(z)=z+\sum_{n=2}^{\infty} b_{n} z^{n}$.

Libera [6] has proved that, for $n \geq 2$,

$$
\left|b_{n}\right| \leq \frac{2(3-2 \gamma)(4-2 \gamma) \ldots(n-2 \gamma)[n(1-\beta)+\beta-\gamma]}{n !},
$$

Now, from relation (2.3), we have

$$
a_{n}=\frac{b_{n}}{1+\alpha(n-1)}
$$

Using this and ( 3.1 ), we obtain the required result

THEOREM 3.2. Let $0<\lambda \leq 1$ and $0 \leq \beta<1$. Let $f$ be defined as

$$
f(z)=\frac{1}{\lambda} z^{1-\frac{1}{\lambda}} \int_{0}^{z} \zeta^{\frac{1}{\lambda}-2} F(\zeta) d \zeta, \quad \frac{1}{\lambda} \geq 1 .
$$

and $F \in Q_{\alpha}[1-2 \beta,-1 ; 1-2 \gamma,-1]$ where $0 \leq \lambda \leq 1, \alpha \geq 0$. Then $f_{\varepsilon} Q_{\alpha}[1-2 \beta,-1 ; 1-2 \gamma,-1]$ PROOF. Let

and let

$$
\begin{aligned}
& F_{1}(z)=(1-\alpha) F(z)+\alpha z F^{\prime}(z), \\
& F_{1}(z)=\frac{1}{\lambda} z^{1-\frac{1}{\lambda}} \int_{0}^{z} \zeta^{\frac{1}{\lambda}-2} F_{1}(\zeta) \mathrm{a} \zeta .
\end{aligned}
$$

Since $F \varepsilon Q_{\alpha}[1-2 \beta,-1,1-2 \gamma,-1]$, it follows from remark 3.2 that $\mathrm{F}_{1} \in \mathrm{K}[1-2 \beta,-1 ; 1-2 \gamma,-1]$. We want to show that $f \in Q_{\alpha}[1-2 \beta,-1 ; 1-2 \gamma,-1]$, where ${ }_{1}(z)=(I-\alpha) f(z)+\alpha f^{\prime}(z)$. Now (3.2) can be written as 


$$
\begin{aligned}
F_{I}(z) & =(1-\alpha) F(z)+\alpha z F^{\prime}(z) \\
& =\alpha z^{2-\frac{1}{\alpha}}\left(z^{\frac{1}{\alpha}-1} F(z)\right) \cdot,
\end{aligned}
$$

and using this, we obtain from (3.3)

$$
\begin{aligned}
f_{1}(z) & =\frac{1}{\lambda} z^{1-\frac{1}{\lambda}} \int_{0}^{z} \zeta^{2-\frac{1}{\alpha}} \zeta^{\frac{1}{\lambda}-2}\left(\zeta^{\frac{1}{\alpha}-1} F(\zeta)\right) \cdot d \zeta \\
& =\frac{\alpha}{\lambda} z^{1-\frac{1}{\lambda}} \int_{0}^{z} \zeta^{\frac{1}{\lambda}-\frac{1}{\alpha}}\left(\zeta^{\frac{1}{\alpha}-1} F(\zeta)\right) \cdot d \zeta
\end{aligned}
$$

So, integrating by parts,

$$
\begin{aligned}
& \left.f_{1}(z)=\frac{\alpha}{\lambda} z^{1-\frac{1}{\lambda}}\left[z^{\frac{1}{\lambda}-\frac{1}{\alpha}}\left(z^{\frac{1}{\alpha}-1} F(z)\right)-\int_{0}^{z}\left(\frac{1}{\lambda}-\frac{1}{\alpha}\right) \zeta^{\frac{1}{\lambda}-\frac{1}{\alpha}-1} F(\zeta)\right) d \zeta\right] \\
& =\frac{\alpha}{\lambda} F(z)+\frac{\alpha}{\lambda}\left(\frac{1}{\alpha}-\frac{1}{\lambda}\right) z^{1-\frac{1}{\lambda}} \int_{0}^{z} \zeta^{\frac{1}{\lambda}-2} F(\zeta) d \zeta \\
& =\alpha\left[\frac{1}{\lambda} F(z)\right]+\alpha\left[\frac{1}{\lambda}\left(1-\frac{1}{\lambda}\right)+\frac{1}{\lambda}\left(\frac{1}{\alpha}-1\right)\right] z^{1-\frac{1}{\lambda}} \int_{0}^{z} \zeta^{\frac{1}{\lambda}-2} F(\zeta) d \zeta \\
& =\alpha z\left[\frac{1}{\lambda} z^{-1} F(z)+\frac{1}{\lambda}\left(1-\frac{1}{\lambda}\right) z^{-\frac{1}{\lambda}} \int_{0}^{z} \zeta^{\frac{1}{\lambda}-2} F(\zeta) d \zeta\right] \\
& +(1-\alpha)\left[\frac{1}{\lambda} z^{1-\frac{1}{\lambda}} \int_{0}^{z} \zeta^{\frac{1}{\lambda}-2} F(\zeta) d \zeta\right] \\
& =\alpha z f^{\prime}(z)+(1-\alpha) f(z) .
\end{aligned}
$$

Now in (3.3) $F_{1} \in K[1-2 \beta,-1 ; 1-2 \gamma,-1]$ and so $f_{1} \in K[1-2 \beta,-1 ; 1-2 \gamma,-1]$, where we have used (2.2) with $\gamma_{1}=\frac{1}{\lambda}-1, A=1-2 \beta, B=-1, C=1-2 \gamma$ and $D=-1$. Thus it follows from remark 3.2 and the relation (3.4) that $f_{\varepsilon Q}[1-2 \beta,-1 ; 1-2 \gamma,-1]$, and this completes the proof.

ACKNOWLEDGEMENT. I am indebted to the referee for helpful comments which improved the exposition of this work.

\section{REFERENCES}

1. NOOR,K.I., and THOMAS,D.K., On quasi-convex univalent functions, Internat.J.Math. \& Math. Sci. 3(1980), 255-266.

2. NOOR,K.I., ON quasi-convex functions and related topics, Int. J.Math. Sci., to appear.

3. NOOR,K.I., and AL-OBOUDI, F.M., Alpha quasi convex univalent functions, Carr. Math. J. 3 (1984), 1-8.

4. JANOWSKI,W., Some external problems for certain families of analytic functions, Ann. Polon. Math. 28(1973), 297-326.

5. SILVIA, E.M., Subclasses of close-to-convex functions, Internat. J. Math. \& Math. Sci. 3 (1983), 449-458.

i. IIBERA,R.J., Some radius of convexity problems, Duke Math.J.(1964), 143-1ui 


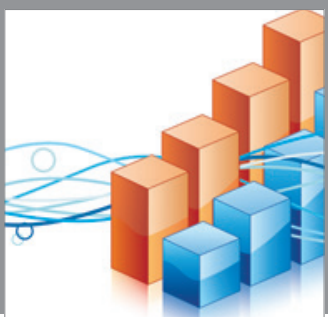

Advances in

Operations Research

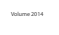

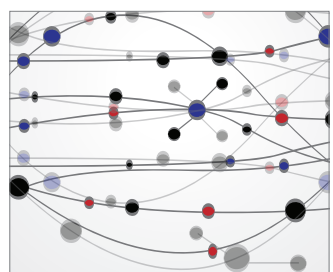

\section{The Scientific} World Journal
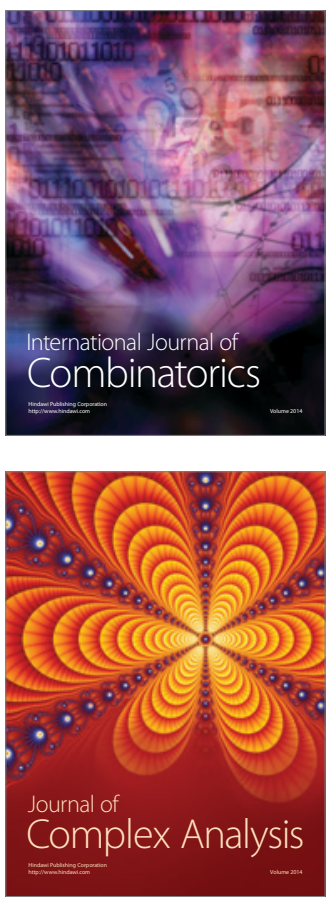

International Journal of

Mathematics and

Mathematical

Sciences
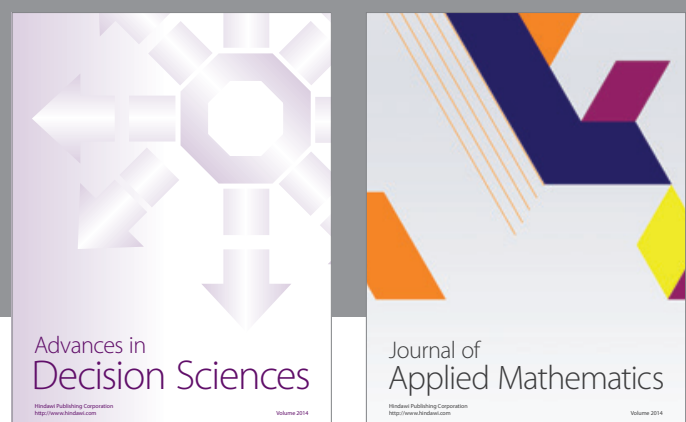

Journal of

Applied Mathematics
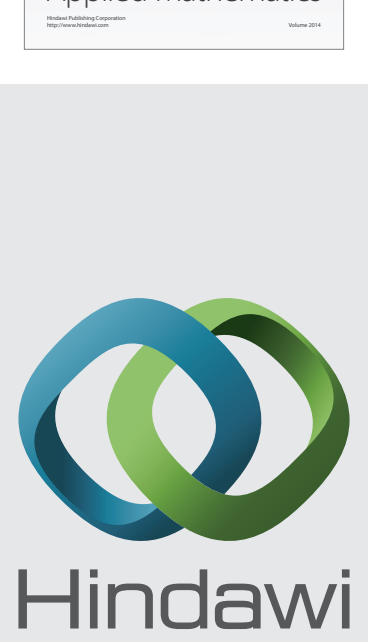

Submit your manuscripts at http://www.hindawi.com
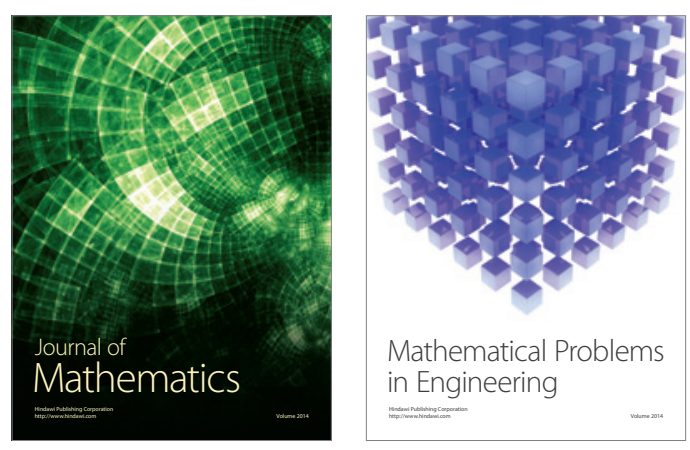

Mathematical Problems in Engineering
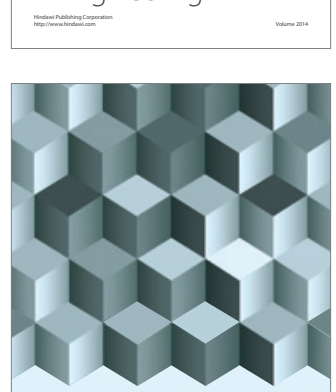

Journal of

Function Spaces
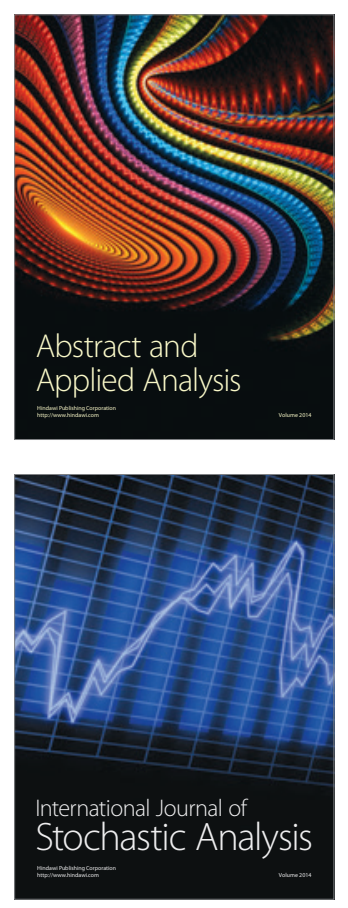

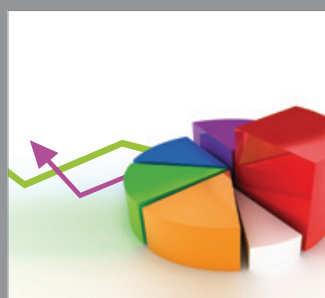

ournal of

Probability and Statistics

Promensencen
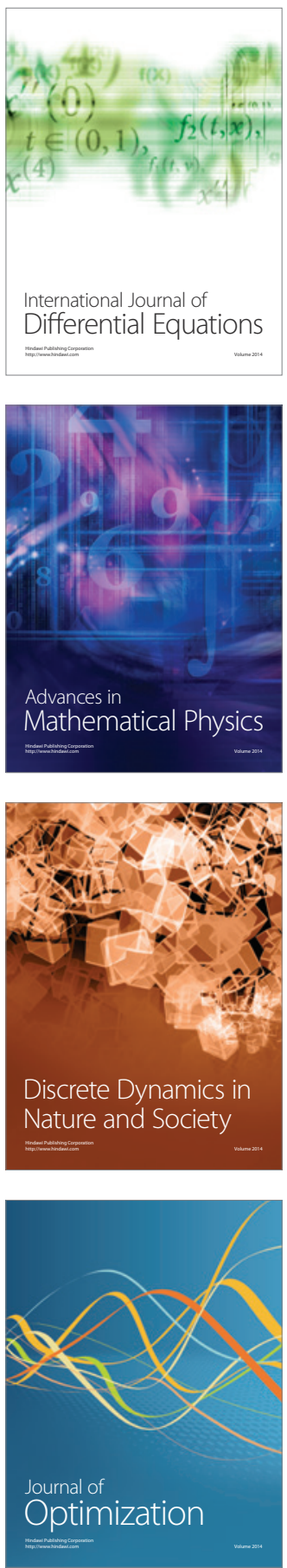